\title{
Blazed gratings on convex substrates for high throughput spectrographs for Earth and Universe observation
}

Frédéric Zamkotsian, Igor Zhurminsky, Patrick Lanzoni, Nicolas Tchoubaklian, Fabian Lütolf, et al.

Frédéric Zamkotsian, Igor Zhurminsky, Patrick Lanzoni, Nicolas Tchoubaklian, Fabian Lütolf, Marc Schnieper, Christian Schneider, Sören Fricke, Marin Fouchier, Myriam Zerrad, Claude Amra, Vincent Costes, Jacques Loesel, "Blazed gratings on convex substrates for high throughput spectrographs for Earth and Universe observation," Proc. SPIE 11852, International Conference on Space Optics - ICSO 2020, 118520N (11 June 2021); doi:

10.1117/12.2599169

SDE Event: International Conference on Space Optics - ICSO 2021, 2021, Online Only 


\section{International Conference on Space Optics-ICSO 2020}

Virtual Conference

30 March-2 April 2021

Edited by Bruno Cugny, Zoran Sodnik, and Nikos Karafolas
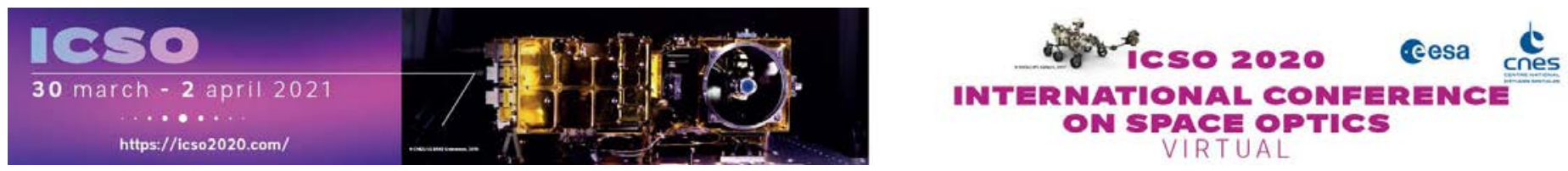

\section{Blazed gratings on convex substrates for high throughput spectrographs for Earth and Universe observation}

\section{Cesa issoperestings lecnes}




\title{
Blazed gratings on convex substrates for high throughput spectrographs for Earth and Universe Observation
}

\author{
Frédéric Zamkotsian ${ }^{1}$, Igor Zhurminsky ${ }^{2}$, Patrick Lanzoni ${ }^{1}$, Nicolas Tchoubaklian ${ }^{1}$, \\ Fabian Lütolf ${ }^{2}$, Marc Schnieper ${ }^{2}$, Christian Schneider ${ }^{2}$, Sören Fricke ${ }^{2}$, \\ Marin Fouchier ${ }^{3,4}$, Myriam Zerrad ${ }^{3}$, Claude Amra $^{3}$ \\ Vincent Costes ${ }^{4}$, Jacques Loesel ${ }^{4}$, \\ ${ }^{1}$ Aix Marseille Univ, CNRS, CNES, LAM (Laboratoire d'Astrophysique de Marseille) UMR 7326, \\ 38 rue F. Joliot Curie, 13388 Marseille Cedex 13, France, \\ ${ }^{2}$ CSEM, Tramstrasse 99, CH-4132 Muttenz, Switzerland \\ ${ }^{3}$ Aix Marseille Univ, CNRS, Centrale Marseille, Institut Fresnel, Facultés des sciences - Campus \\ Saint Jérôme, Avenue Escadrille Normandie-Niemen, 13397 Marseille, France \\ ${ }^{4}$ CNES, 18 Avenue Edouard Belin, 31401 Toulouse Cedex 9, France \\ e-mail address: frederic.zamkotsian@lam.fr
}

\begin{abstract}
In next generation space instrumentation for Earth and Universe Observation, new instrument concepts include often non planar gratings. Their realization is complex and costly. We propose a new technology for designing and realizing convex blazed gratings for high throughput spectrographs.

For this purpose, our requirements are driven by a Digital-Micromirror-Device-based (DMD) MOS instrument to be mounted on the Telescopio Nazionale Galileo (TNG) and called BATMAN. The two-arm instrument is providing in parallel imaging and spectroscopic capabilities. The objects/field selector is a 2048 x 1080 micromirrors DMD, placed at the focal plane of the telescope; it is used as a programmable multi-slit mask at the entrance of the spectrograph. The compact Offner-type spectrograph design contains a low density convex grating to disperse light. For optimization of the spectrograph efficiency, this convex grating must be blazed.

A blazed reflective grating has been designed with a period of $3300 \mathrm{~nm}$ and a blaze angle of $5.04^{\circ}$, and fabricated into convex substrates with $225 \mathrm{~mm}$ radius of curvature and a footprint diameter of $63.5 \mathrm{~mm}$. The blaze is optimized for the center wavelength of $580 \mathrm{~nm}$ within the spectral range of $400-800 \mathrm{~nm}$. Such gratings have been fabricated and coated with a silver-based layer, with a final $7^{\circ}$ blaze angle over the whole surface. Efficiency close to $90 \%$ on the 1 st diffraction order at $700 \mathrm{~nm}$ has been obtained, measured on BATMAN spectroscopic arm. Detailed mapping of the blazed grating showed a very good period uniformity with up to $0.5 \%$ deviation. Grating depth and blaze angle have higher deviation, up to 7\%. An optimized device with the exact required blaze angle would reach the same efficiency and be centered on the mid of $400-800 \mathrm{~nm}$ wavelength band: its realization is on-going.

The grating brings a significant contribution in the total amount of straylight at instrument level. Their straylight level remains a critical issue, and its reduction by specific and controlled implementation of improvements in manufacturing process is a challenge to tackle. Preliminary straylight measurement has been done and shows a lowest straylight level below $10^{-2} \mathrm{sr}^{-1}$ between the diffraction orders.

This new type of non-planar reflective gratings will be the key component for future high throughput spectrographs in space missions.
\end{abstract}

Key words: non-planar grating, convex blazed grating, spectro-imager, multi-object spectrograph, astronomical instrumentation, MOEMS.

\section{INTRODUCTION}

In next generation space instrumentation for Earth Observation, new instrument concepts (hyper-spectral imaging and spectroscopy) must be compact, highly efficient and versatile. MOEMS devices may provide new observational modes as generating a smart-slit at the entrance of the spectrograph for removing clouds and bright objects (and then decrease greatly the straylight in the instrument), or be the key component of wide field programmable spectrographs. ${ }^{1}$ These new instrument concepts include often non planar gratings. 
Next-generation infrared astronomical instrumentation for ground-based and space telescopes could be based on MOEMS programmable slit masks for multi-object spectroscopy (MOS). MOS is used extensively to investigate astronomical objects optimizing the Signal-to-Noise Ratio (SNR): high precision spectra are obtained and the problem of spectral confusion and background level occurring in slitless spectroscopy is cancelled. Fainter limiting fluxes are reached and the scientific return is maximized both in cosmology, in galaxies formation and evolution, in stellar physics and in solar system small bodies characterization.

Major telescopes around the world are equipped with MOS in order to simultaneously record several hundred spectra in a single observation run. Next generation MOS for space like the Near Infrared Multi-Object Spectrograph (NIRSpec) for the James Webb Space Telescope (JWST) will use a programmable multi-slit mask. MOEMS programmable slit masks would be next-generation devices for selecting objects. The programmable multi-slit mask requires remote control of the multi-slit configuration in real time. During the early-phase studies of the European Space Agency (ESA) EUCLID mission, a MOS instrument based on a MOEMS device has been assessed. Due to complexity and cost reasons, slitless spectroscopy was chosen for EUCLID, despite a much higher efficiency with slit spectroscopy.

MOEMS devices such as micromirror arrays (MMA) ${ }^{2,3,4}$ or micro-shutter arrays (MSA) ${ }^{5}$ are promising solutions. MMAs are designed for generating reflective slits, while MSAs generate transmissive slits. In Europe an effort is currently under way to develop single-crystalline silicon micromirror arrays for future generation infrared multi-object spectroscopy (collaboration LAM / EPFL-CSEM). ${ }^{6,7}$ By placing the programmable slit mask in the focal plane of the telescope, the light from selected objects is directed toward the spectrograph, while the light from other objects and from the sky background is blocked. To get more than 2 millions independent micromirrors, the only available component is a Digital Micromirror Device (DMD) chip from Texas Instruments (TI) that features 2048 x 1080 mirrors and a $13.68 \mu \mathrm{m}$ pixel pitch. DMDs have been tested in space environment $\left(-40^{\circ} \mathrm{C}\right.$, vacuum, radiations) by LAM and no showstopper has been revealed. ${ }^{8}$

We are developing a 2048 × 1080 Digital-Micromirror-Device-based (DMD) MOS instrument to be mounted on the 3.6m Telescopio Nazionale Galileo (TNG) and called BATMAN. ${ }^{9}$ A two-arm instrument has been designed for providing in parallel imaging and spectroscopic capabilities. BATMAN will be mounted on the folded Nasmyth platform of TNG. Thanks to its compact design, high throughput is expected. The two arms with F/4 on the DMD are mounted on a common bench, and an upper bench supports the detectors thanks to two independent hexapods. The stiffness of the instrument is guaranteed thanks to a box architecture linking both benches. ${ }^{10}$ The volume of BATMAN is $1.4 \times 1.2 \times 0.75 \mathrm{~m}^{3}$, with a total mass of $400 \mathrm{~kg}$. Mounting of all sub-systems has been done and integration of the individual arms is under way. BATMAN on the sky is of prime importance for characterizing the actual performance of this new family of MOS instruments, as well as investigating the new operational procedures on astronomical objects (combining MOS and IFU modes, different spatial and spectral resolutions in the same FOV, absolute (spectro-) photometry by combining imaging and spectroscopy in the same instrument, automatic detection of transients ...).

This instrument will be placed at TNG by 2022. ${ }^{11}$ BATMAN concept has also been proposed for a space mission as BATMAN flies. ${ }^{12}$ The BATMAN compact Offner-type spectrograph design contains a low density convex grating to disperse light. In order to optimize the spectrograph efficiency, this convex grating must be blazed at the right angle for maximizing the light in the first order of diffraction.

For Earth Observation, a good example is the CHIME mission devoted to provide routine hyperspectral observations through the Copernicus Programme in support of EU and related policies, for the management of natural resources, assets and benefits. This unique visible -to- shortwave infrared spectroscopy based observational capability will in particular support new and enhanced services for food security, sustainable agriculture and raw materials. This includes biodiversity management, soil properties characterization, sustainable mining practices and environment preservation. CHIME is then a complex push-broom imaging spectrometer with spectral range $400 \mathrm{~nm}-2500 \mathrm{~nm}$ and resolution $10 \mathrm{~nm}$. At LAM, we are involved in the conception of new MOEMS-based spectro-imager for Earth observation. We have designed an innovative spectro-imager, with constraints of wide $2 \mathrm{D}$ field of view $\left(3^{\circ} \times 1^{\circ}\right)$, image quality $(<2$ pixels $=11 \mu \mathrm{m})$ and compacity. The instrument is panchromatic with a medium spectral resolution between 1000 and 2000, fitting in a $40 \mathrm{~cm}$ x $50 \mathrm{~cm}$ x $90 \mathrm{~cm}$ box. A 3-mirror solution for both imaging and spectrograph arms has been designed, using only aspheric surfaces thus allowing for easier alignment and tolerancing. ${ }^{13}$

This paper describes the design, the realization and the characterization of a new type of non-planar reflective gratings, key components for future high throughput spectrographs in space missions. 


\section{CONVEX BLAZED GRATING PARAMETERS}

BATMAN is a compact spectro-imager with two arms in parallel: a spectroscopic channel and an imaging channel. Both arms are fed by using the two DMD mirrors stable positions (Fig. 1). ${ }^{9}$

The compact Offner-type spectrograph design contains a low density convex grating (SGR in Fig. 1) to disperse light. In order to optimize the spectrograph efficiency, this convex grating must be blazed at the right angle for maximizing the light in the first order of diffraction.

The grating requirements are:

- $\quad$ Convex substrate

- $\quad$ Radius of curvature $225 \mathrm{~mm}$

- $\quad$ Diameter $63 \mathrm{~mm}$

- $\quad$ Number of lines: $3001 / \mathrm{mm}$

- $\quad$ Wavelength range 400-800 nm

- $\quad$ Center wavelength $580 \mathrm{~nm}$

- $\quad$ Blaze angle $5.04^{\circ}$

- $\quad$ Incidence angle (in-plane) $24^{\circ}$

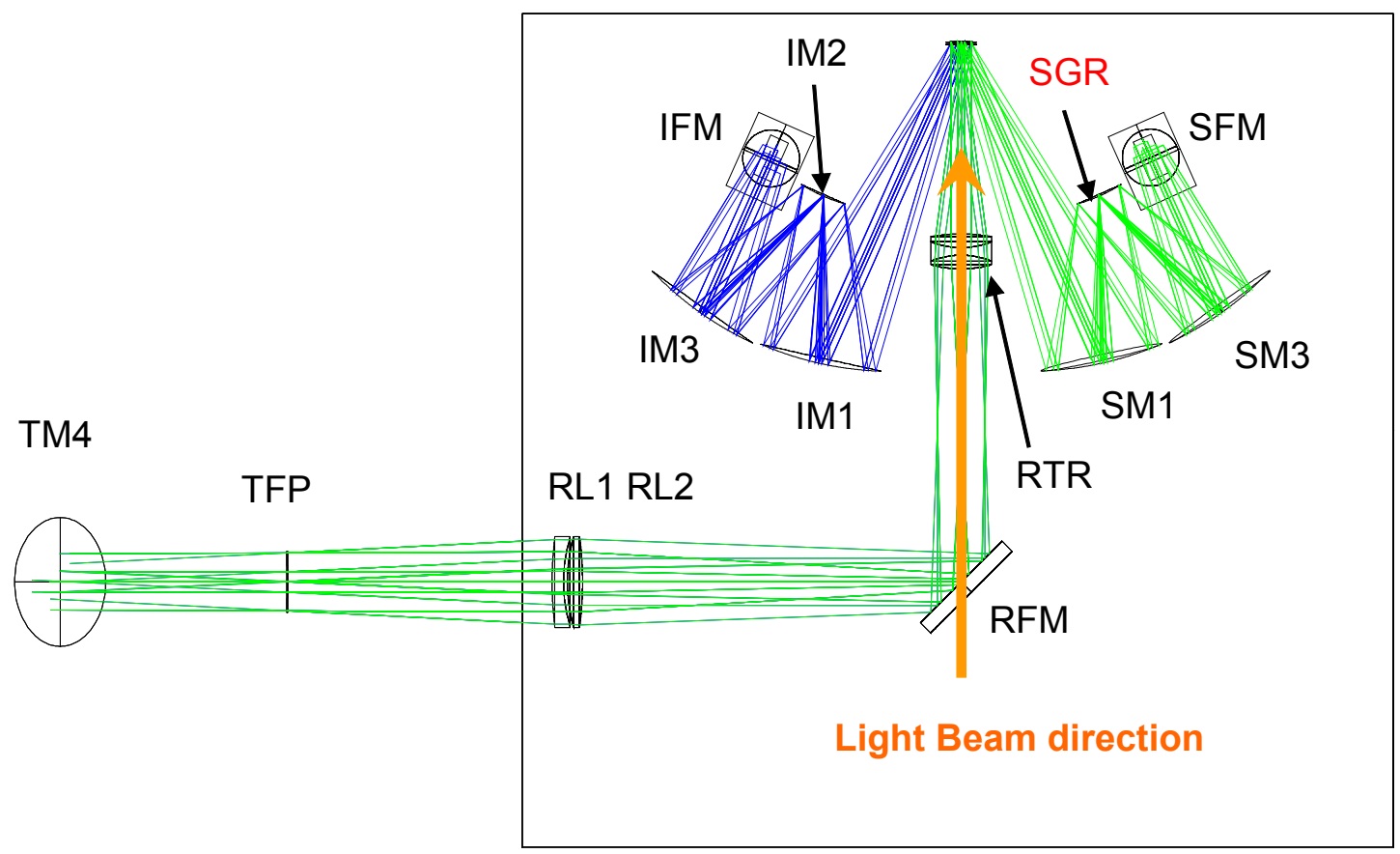

Fig. 1: Principle of BATMAN spectro-imager

The substrate requirements are:

- $\quad$ Quartz / Silicon Oxyde

- $\quad \lambda / 4$ surface quality

- $\quad$ Grating pattern etched in substrate

The coating requirements are:

- $\quad$ Protected silver

- $\quad$ Conformal deposition on grating surface 


\section{CONVEX BLAZED GRATING REALIZATION}

The description of the process to realize the master plane blazed grating is shown in Fig. 2.

The master of the blazed grating structure has been originated on a flat substrate starting from a rectangular grating with a period of $3300 \mathrm{~nm}$. The rectangular grating was UV replicated twice using Sol-Gel material and subsequently converted into a blazed shape by angular Ar ion etching. The desired blazed grating parameters like depth and blaze angle have been reached by adjusting the initial grating depth in Sol-Gel as well as the Ar etching angle and duration.

The transfer of the blazed grating from a flat surface onto a convex substrate needs a flexible support. A flexible stamp was generated by UV replication of the blazed grating, utilizing a flexible nanoimprint material (Fig. 3).

- Step 1: $1^{\text {st }}$ Replication.
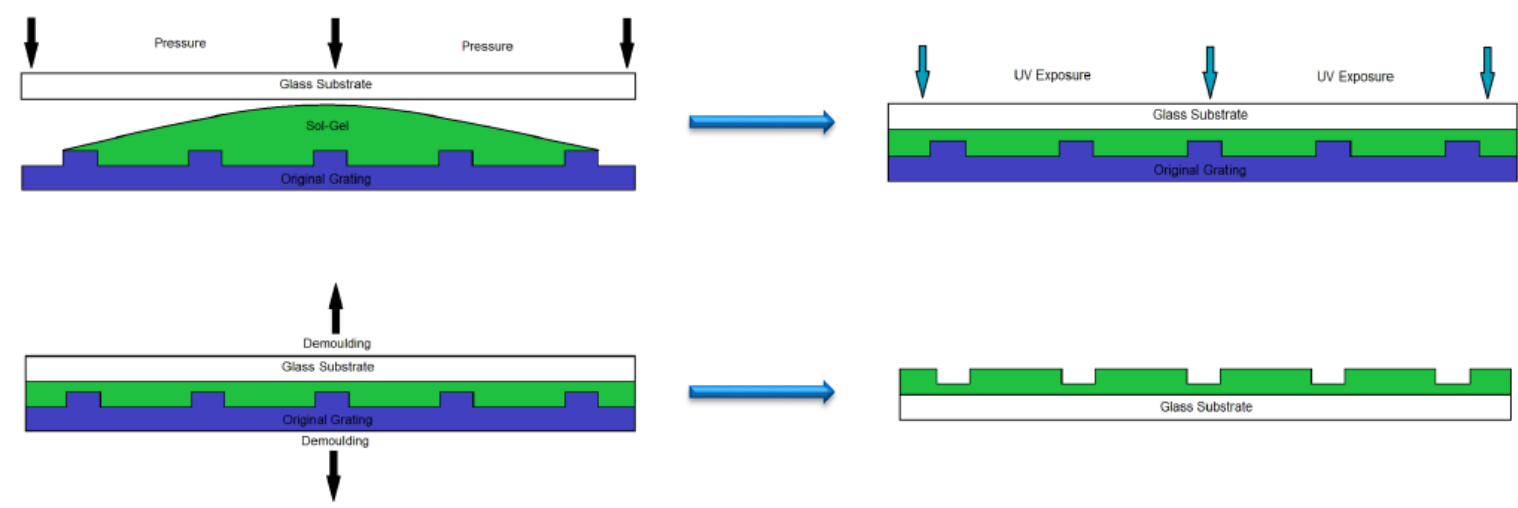

Fig. 2: Convex blazed grating realization (step 1)

- Step 2: $2^{\text {nd }}$ replication

- Step 3: Ar angle etching

- Step 4: Flexible stamp
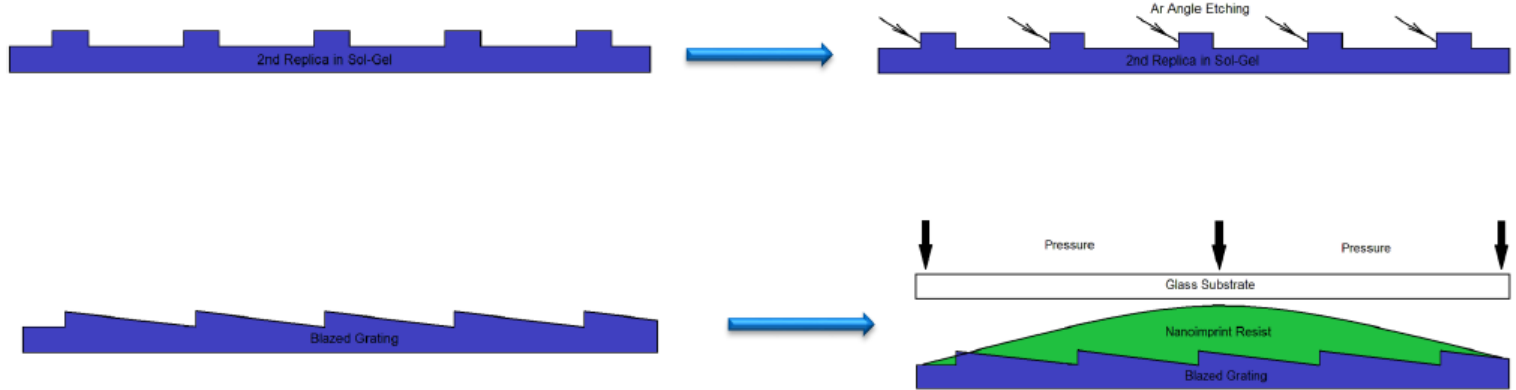

Fig. 3: Convex blazed grating realization (steps 2, 3, 4)

The blazed grating is then transferred from a flat surface into a convex substrate. The flexible stamp was used to emboss a thin layer of Sol-Gel, spin-coated on the convex substrate. The nanoimprint material is used as a masking layer for Reactive Ion Etching of the convex substrate. After curing, the structure was transferred into the substrate by etching. With this approach, the final component is a convex substrate with the grating structure etched into the volume. The monolithic approach is considered more preferable due to the absence of a quartz/silicon-oxide substrate to Sol-Gel interface prone to fatigue, perfectly suited for space environment (Fig. 4). 


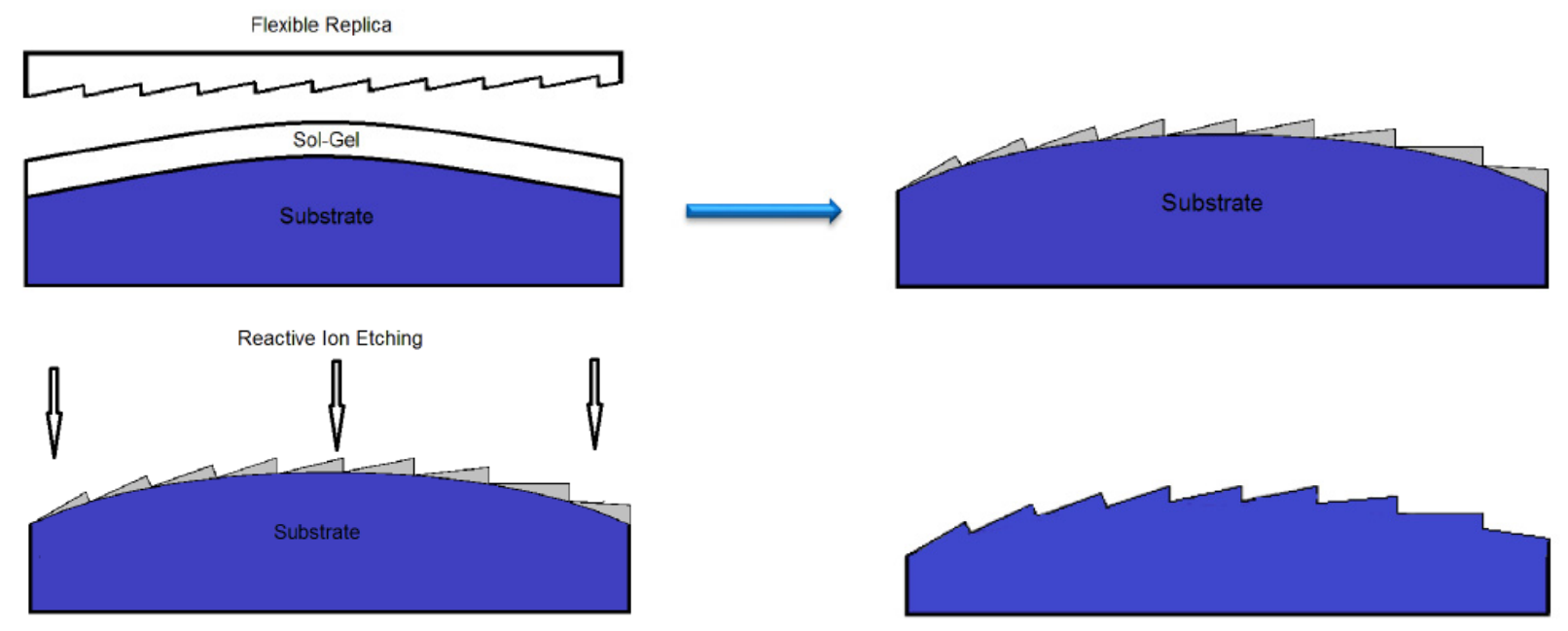

Fig. 4: Blazed Grating Transfer into a Convex Substrate

This approach has been successful and a grating has been fabricated. Its surface is characterized by Scanning Electron Beam Microscope: in Fig. 5 are shown a general view and the profile of the realized grating. The grating coating is protected silver from Optics BALZERS, identical to other BATMAN mirrors coating. The reflectivity is over $98 \%$ over the whole wavelength range $(400-800 \mathrm{~nm}) .{ }^{14}$

The blazed grating transferred into the convex substrate exhibits very smooth lines and a grating profile with $7^{\circ}$ blaze angle (with respect to the expected $5.04^{\circ}$ ) over the whole surface. All grooves are perfectly defined with a smooth surface (minimizing the straylight of the component).

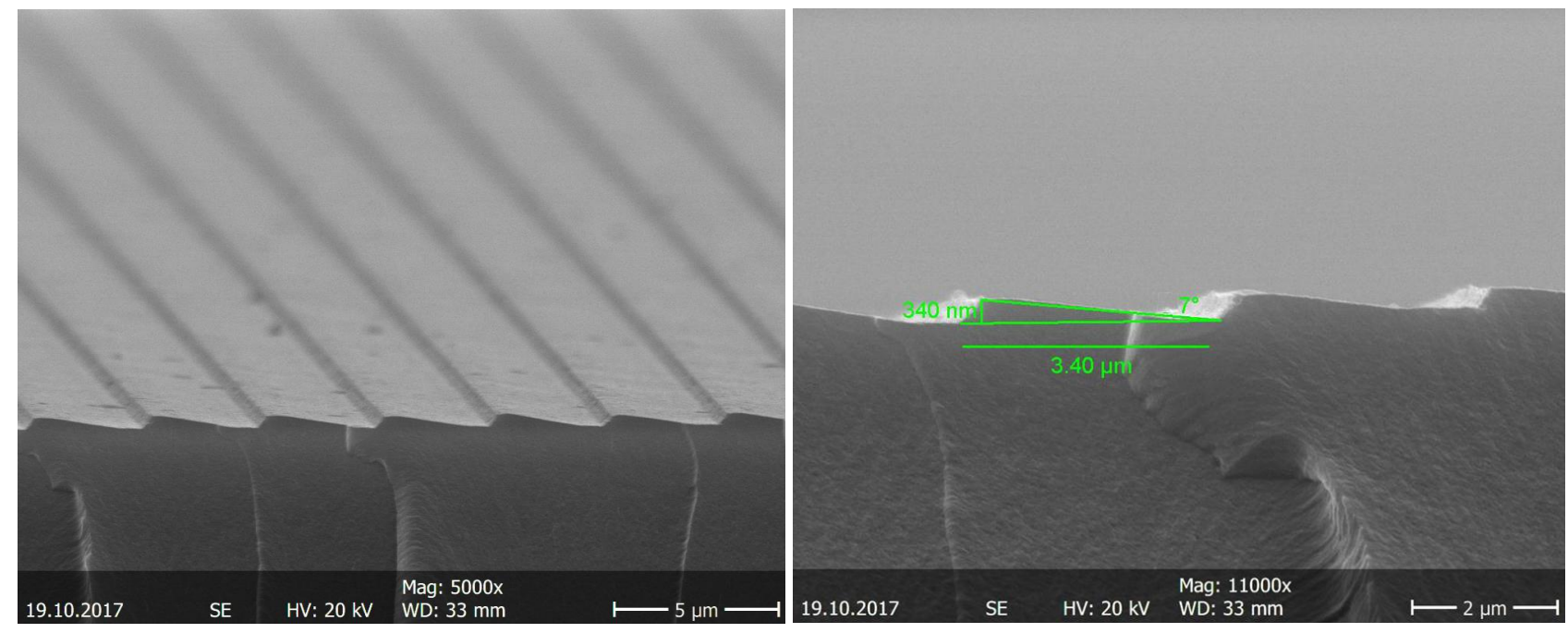

Fig. 5: Blazed Grating Transfer into a Convex Substrate: a final figure of $7^{\circ}$ blaze angle has been obtained on the whole surface, very close to the required value; general view and profile

Detailed mapping of the blazed grating showed a very good period uniformity with up to $0.5 \%$ deviation. Grating depth and blaze angle have higher deviation, up to $7 \%$. 


\section{CONVEX BLAZED GRATING CHARACTERIZATION}

Characterizing a convex blazed grating requires an optical set-up able to collimate the optical beam on the convex surface and then to image the entrance slit on a detector. This complex system is actually equivalent to align one arm of BATMAN (see Fig. 1). Within this instrumental project, we have currently received all optics (mirrors and lenses) as well as all opto-mechanical mounts to hold the optical components. BATMAN integration has started by assembling all sub-systems on a Newport damped table in order to review both the integration procedure as well as a first performance evaluation (Fig. 6). The blazed grating efficiency measurement takes place in this process.

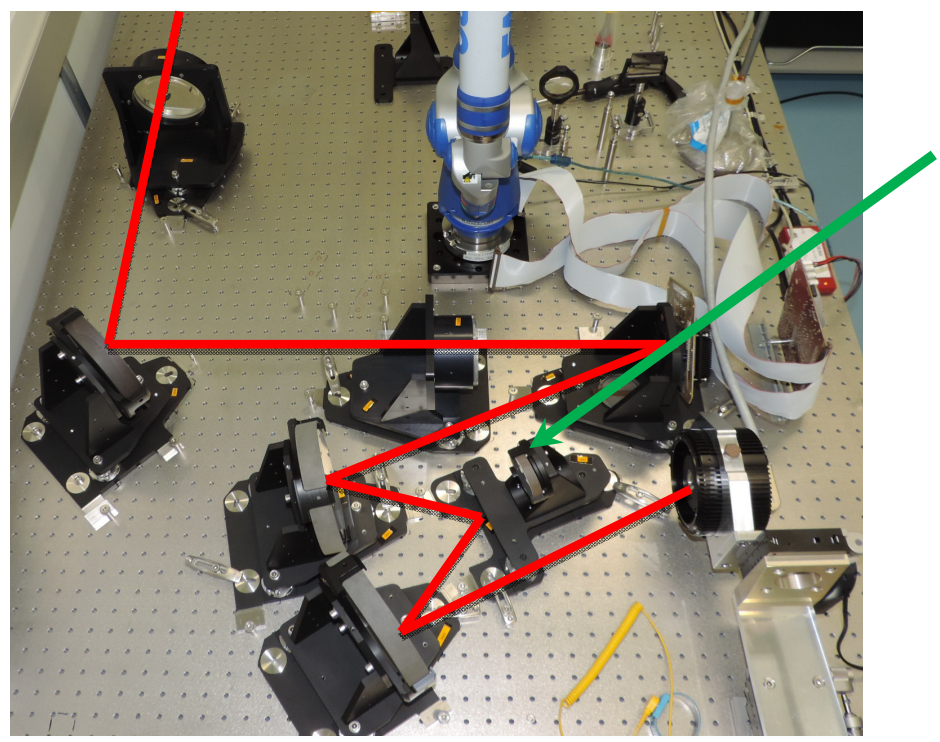

\section{Mirror or Blazed grating}

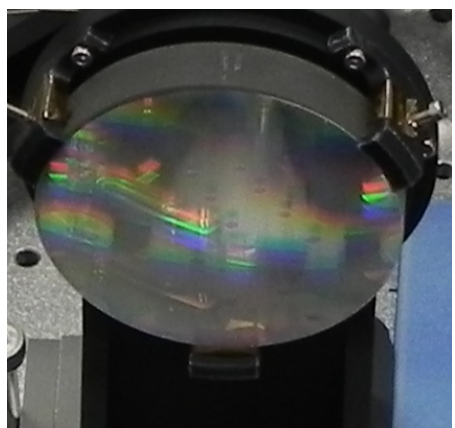

Fig. 6: Blazed convex grating characterization set-up, i.e. BATMAN spectro/imaging arm; the light path is marked in red from top (instrument entrance) to bottom (detector) close-view of the realized blazed grating

The input beam is issued from a fibered white light source with a nearly flat response in the visible range. A wavelength selection module is inserted at the output of the source in order to select any wavelength with $1 \mathrm{~nm}$ wavelength bandwidth. The source is then tunable from $460 \mathrm{~nm}$ up to $700 \mathrm{~nm}$; the output power could be also tuned. The fibre is then placed at the location of the entrance slit of the spectrograph, i.e. at the DMD centre location. The monomode fibre defines then a point source at the entrance of the spectrograph.

The overall grating efficiency has been obtained for the grating inserted in its proper location within the instrument, by measuring the throughput of the set-up while the wavelength is tuned from 460 to $700 \mathrm{~nm}$; these values are weighted by the throughput of the instrument with a convex mirror replacing the convex grating. The measured response (red crosses) is presented in Fig. 7: an efficiency close to $90 \%$ on the 1st diffraction order is obtained at $700 \mathrm{~nm}$.

At short wavelength $(450-500 \mathrm{~nm})$, the efficiency is rather low, leading to the fact that the central wavelength of the component has to be shifted to a shorter wavelength for a better balance of the efficiency within the entire wavelength range.

Simulated grating efficiency with $7^{\circ}$ blaze angle for TE, TM and Non-Polarized (NP) beams for [400nm-800nm] wavelength range is superimposed with the measured values in Fig. 7. Good agreement between the simulation and the measurement confirms the $7^{\circ}$ blaze angle of the device.

An optimized device with the exact required blaze angle $\left(5.04^{\circ}\right)$, would reach the same efficiency $(>\mathbf{9 0 \%}$ at peak wavelength) and be centered on the mid of $400-800 \mathrm{~nm}$ wavelength band (Fig. 8). Simulated responses for TE, TM and Non-Polarized (NP) beams are presented with very close values at short wavelengths, but there are some discrepancies at larger wavelengths. 


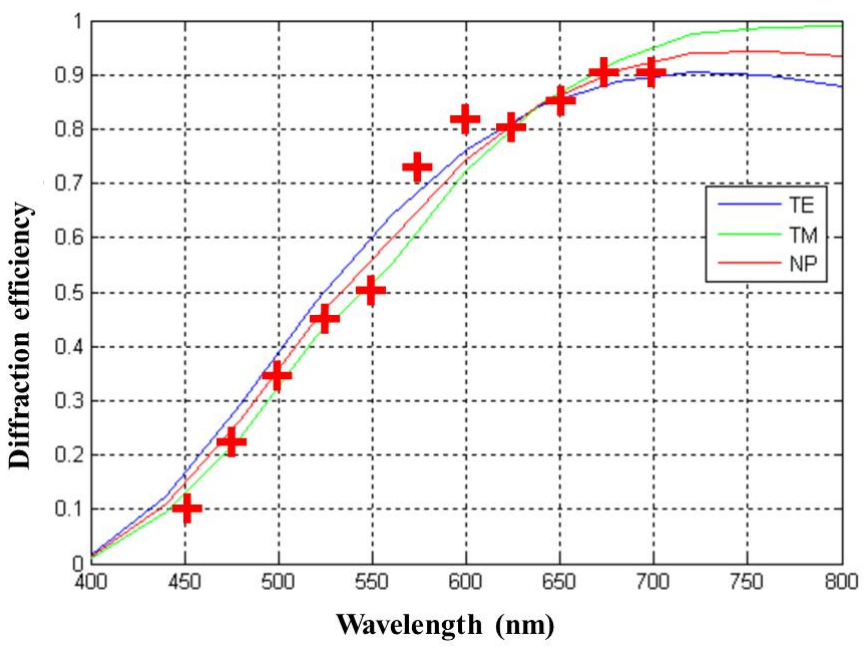

Fig. 7: Blazed convex grating efficiency from $460 \mathrm{~nm}$ to $700 \mathrm{~nm}$; measured values are displayed with red crosses; Simulated grating efficiency with $7^{\circ}$ blaze angle for TE, TM and Non-Polarized (NP) beams for [400nm-800nm] wavelength range.

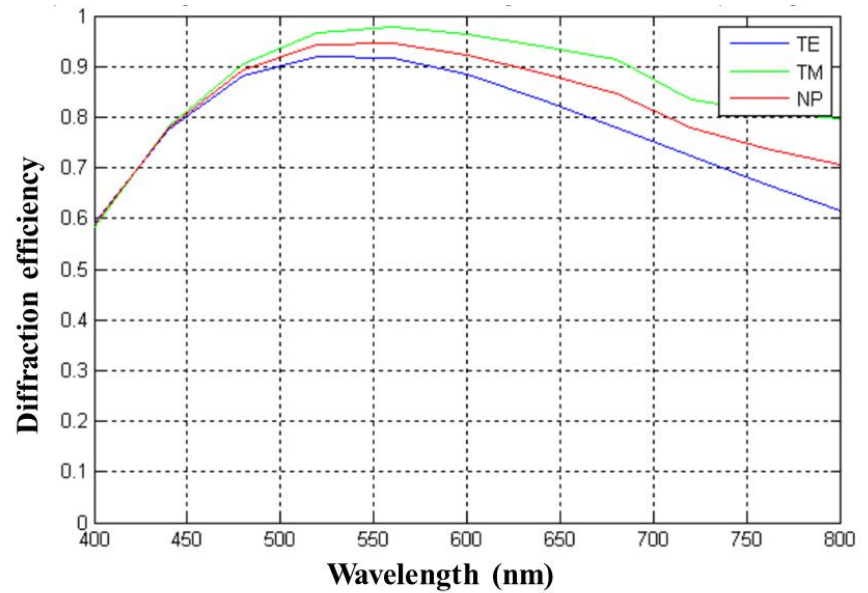

Fig. 8: Optimized blazed convex grating efficiency with $5.04^{\circ}$ blaze angle, centered at $580 \mathrm{~nm}$ for $[400 \mathrm{~nm}-800 \mathrm{~nm}]$ wavelength range. Simulated responses for TE, TM and Non-Polarized (NP) beams.

The next steps for the characterization of the blazed convex grating are the measurement of the efficiency for the complete wavelength range from 400 to $800 \mathrm{~nm}$, and the evaluation of the dependence of the efficiency with respect to the polarization. Preliminary straylight measurement is presented in the next paragraph.

This new technology is versatile for a wide parameters range, and, as the grating is etched into the substrate, this component is compatible for space applications. Space qualification campaign is then foreseen.

\section{STAYLIGHT MEASUREMENT}

Missions involving spectral instruments have stringent spectral and radiometric requirements. To achieve them, the physical straylight inside the instrument must be minimized. Additional improvements are performed by applying a postprocessing, but the complete processing is so complex, that it is mandatory to obtain raw images of very good quality. When a spectral instrument is well designed, its straylight performance is limited by technological limitations, such as the residual roughness or particulate contamination. In this context, the grating brings a significant contribution in the total amount of straylight at instrument level. Despite advances in the design and manufacturing techniques of diffraction gratings, their straylight level remains a critical issue. Its reduction by specific and controlled implementation of improvements in manufacturing process is a challenge to tackle. 
This roughness can be divided in 3 categories: the Line Edge Roughness (LER), the Line Width Roughness (LWR), the Side Wall Roughness (SWR). The Bidirectional Scattering Distribution Function (BSDF) can then be calculated for given conditions of illuminations (wavelength range, polarization, angular aperture, angle of incidence...).

In terms of optical metrology, BSDF patterns, diffraction efficiency and polarization sensitivity are measured locally with the instrument SALSA (Spectral and Angular Light Scattering characterization Apparatus) developed by the Light scattering group of the Institut Fresnel. ${ }^{15,16}$

The values of resolution and step size of the scatter angle as well as the wavelengths are fixed by the numerical analysis. SALSA nominal angular resolution can be fixed to $0.1^{\circ}$, and close to specular analysis can be done until $10-3^{\circ}$ if necessary. The wavelengths of measurement can be arbitrarily chosen on the spectral range [450 nm -1650 nm] with a spectral resolution of $0.1 \mathrm{~nm}$. On the whole spectral range of validity [450 nm $-1650 \mathrm{~nm}$ ], the detection is limited by Rayleigh scattering of air particles, which is close to $10^{-8} \mathrm{str}^{-1}$ and the accuracy of any measurement point is better than 1\%. SALSA is in clean room ISO 6 at the Institut Fresnel and the set-up is fully automatized to allow very long measurements. The illumination is collimated with an angular aperture of $0.1^{\circ}$ (it can be tuned until $10^{-3}$ in the visible range) to simulate the illumination with a plane wave and optimize the comparison between metrology and modelling. The number of spatial positions on the grating (illumination spots) is fixed to be representative of the homogeneity of the tested components.

The convex blazed grating under development fits well with the applications such as OLCI or CHIME. Such applications become very attractive all over the world, and certainly have an interesting future. The different sources of straylight are resumed in table 1, together with the grating realization process steps, and the potential source of issues.

\begin{tabular}{|c|c|c|}
\hline Source of straylight & Associated process step & Potential source of issue \\
\hline Depth inhomogeneity & Dry etching into substrate & Inhomogeneous dry etching \\
\hline $\begin{array}{c}\text { Blaze/anti-blaze angle } \\
\text { inhomogeneity }\end{array}$ & Transfer & $\begin{array}{c}\text { Inhomogeneous transfer into } \\
\text { nanoimprint material }\end{array}$ \\
\cline { 2 - 3 } & Dry etching into substrate & Inhomogeneous dry etching \\
\hline Line edge roughness & Photolithography & Local variation of exposure \\
\cline { 2 - 3 } & Chromium mask & Chromium film roughness \\
\hline $\begin{array}{c}\text { Blaze, anti-blaze surface } \\
\text { roughness }\end{array}$ & $\begin{array}{c}\text { Ar angle etching, imprint, } \\
\text { reactive ion etching }\end{array}$ & $\begin{array}{c}\text { Isotropy of etching method } \\
\text { + flexible stamp roughness }\end{array}$ \\
\hline
\end{tabular}

Table 1: Different sources of straylight, associated with the grating realization process steps and the potential source of issues.

Figure 9 shows the convex blazed grating installed in the SALSA instrument during the straylight measurement.

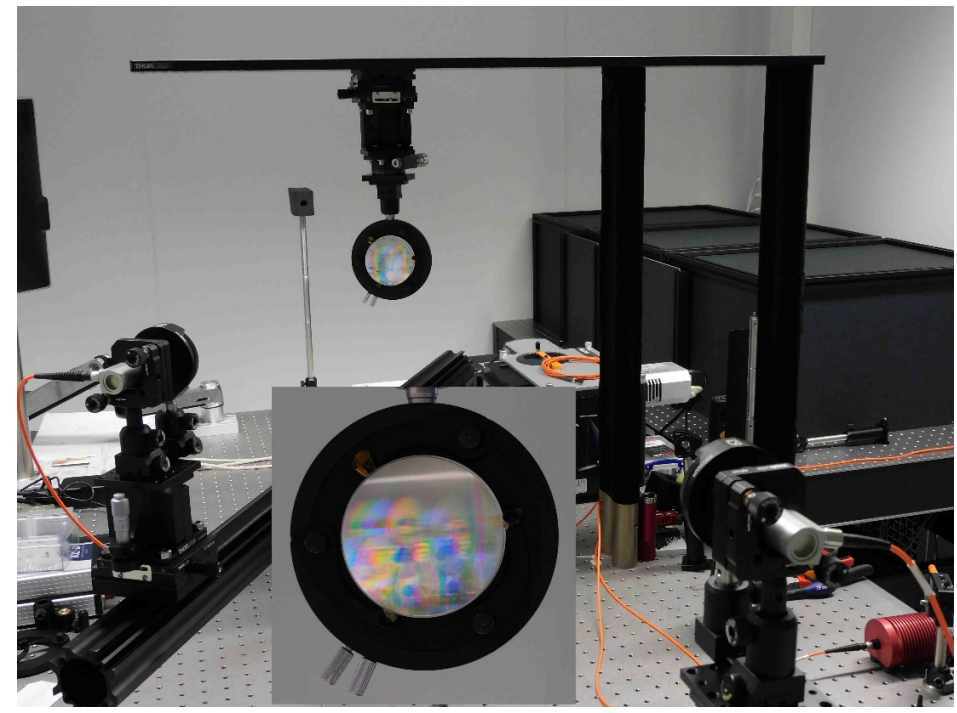

Fig. 9: Convex blazed grating during the measurement in the SALSA instrument Insert: a largest view of the grating. 
Figure 10 shows the result of the BSDF measurement of the convex blazed grating device at 2 wavelengths of illumination: $650 \mathrm{~nm}$ and $700 \mathrm{~nm}$. The measurement has been performed with SALSA instrument under oblique incidence with unpolarized illumination.

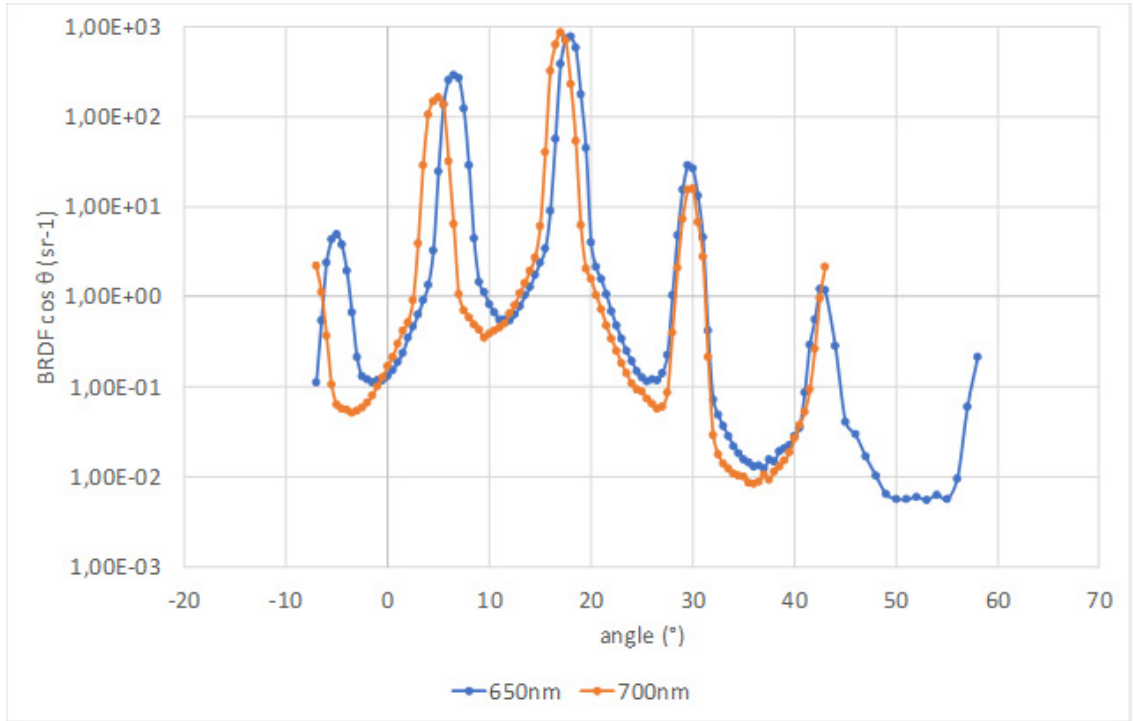

Fig. 10: BSDF measurement of the convex blazed grating device under oblique incidence $\left(30^{\circ}\right)$ at 650 and $700 \mathrm{~nm}$ performed with SALSA instrument

The $0^{\text {th }}$ order is clearly marked at $30^{\circ}$ angle where both wavelengths diffraction peaks are superimposed. To the left $1^{\text {st }}$, $2^{\text {nd }}$ and $3^{\text {rd }}$ orders are clearly revealed with a spectral dispersion increasing with the order number; to the right of the $0^{\text {th }}$ order, -1 order is measured. The highest efficiency is on the $1^{\text {st }}$ order of diffraction.

This first measurement performed on the convex blazed grating shows a lowest straylight level below $10^{-2} \mathbf{s r}^{-1}$ between the diffraction orders.

The width of diffraction orders is quite large, this can be explained by the diameter of the probing spot (4 mm) in the nominal configuration of SALSA. Due to its convexity, the grating is thus illuminated under several angles of incidence with a single beam. The resulting measurement is the convolution of this angular aperture with the diffraction orders. This will be corrected by changing the diameter of the illumination spot and by considering the spreading of the diffraction orders induced by convexity in the modeling. Thus, we can extract from the measurement the BRDF between the diffraction orders and link it to the structural parameters of the gratings.

It is also worth noting that no ghost is present in the measured BSDF so that development of the optimal grating is expected to focus on reduction of scattering. Potential improvement on the manufacturing to reduce this value will be evaluated, tested and applied to the optimized device. 


\section{CONCLUSION}

In next generation space instrumentation for Earth and Universe Observation, new instrument concepts will include nonplanar gratings. We have proposed a new technology for designing and realizing convex blazed gratings for high throughput spectrographs.

The designed grating corresponds to the optical component needed for BATMAN spectro-imager instrument. This blazed reflective grating has a period of $3300 \mathrm{~nm}$ and a blaze angle of $5.04^{\circ}$. It has been successfully fabricated into convex substrates with $225 \mathrm{~mm}$ radius of curvature and a footprint diameter of $63.5 \mathrm{~mm}$. The blaze is optimized for the center wavelength of $580 \mathrm{~nm}$ within the spectral range of $400-800 \mathrm{~nm}$. Convex blazed gratings have been fabricated and coated with protected silver, with a final $7^{\circ}$ blaze angle over the whole surface. Performance characterization shows an efficiency close to $90 \%$ on the 1 st diffraction order at $700 \mathrm{~nm}$. An optimized device with the exact required blaze angle, would reach the same efficiency and be centered on the mid of 400-800nm wavelength band. Preliminary straylight measurement has been done and shows a lowest straylight level below $10^{-2} \mathrm{sr}^{-1}$ between the diffraction orders.

This new technology is versatile for a wide parameters range, and, as the grating is etched into the substrate, this component is compatible for space applications

The first instrument demonstration on-sky of this new grating will be done on board BATMAN mounted on the Telescopio Nazionale Galileo by 2022.

This new type of non-planar reflective gratings will be the key component for next generation compact and highly efficient spectrographs.in space missions, for Universe Observation, Earth Observation and Planetology.

\section{ACKNOWLEDGEMENT}

The authors would like to thank the French Space Agency (Centre National d'Etudes Spatiales, CNES) for partly funding this work, and Jacques Berthon (from CNES) for fruitful discussions during the project.

\section{REFERENCES}

[1] Frederic Zamkotsian, Arnaud Liotard, Patrick Lanzoni, T. Viard, " Optical MEMS in space instruments for Earth Observation and Astronomy", Proceedings of the SPIE conference on MOEMS 2013, Proc. SPIE 8616, San Francisco, USA, Février 2013

[2] R. Burg, P.Y. Bely, B. Woodruff, J. MacKenty, M. Stiavelli, S. Casertano, C. McCreight and A. Hoffman, "Yardstick integrated science instrument module concept for NGST", in Proceedings of the SPIE conference on Space Telescope and Instruments V, SPIE 3356, 98-105, Kona, Hawaii, (1998)

[3] F. Zamkotsian, K. Dohlen, D. Burgarella, V. Buat, "Aspects of MMA for MOS: optical modeling and surface characterization, spectrograph optical design", in Proceedings of the NASA conference on "NGST Science and Technology Exposition", ASP Conf. Ser. 207, 218-224, Hyannis, USA, (1999)

[4] M. Robberto, A. Cimatti, A. Jacobsen, F. Zamkotsian, F. M. Zerbi, "Applications of DMDs for Astrophysical Research", in Proceedings of the SPIE conference on MOEMS 2009, Proc. SPIE 7210, San Jose, USA, (2009)

[5] M. J. Li; A. D. Brown; A. S. Kutyrev; H. S. Moseley; V. Mikula, " JWST microshutter array system and beyond", Proc. SPIE 7594, San Francisco, USA (2010)

[6] S. Waldis, F. Zamkotsian, P. Lanzoni, W. Noell, N. de Rooij, "Micromirrors for multiobject spectroscopy: optical and cryogenic characterization", in Proceedings of the SPIE conference on MOEMS 2008, Proc. SPIE 6887, San Jose, USA (2008)

[7] M. Canonica, F. Zamkotsian, P. Lanzoni, W. Noell, N. de Rooij, "The two-dimensional array of 2048 tilting micromirrors for astronomical spectroscopy," Journal of Micromechanics and Microengineering, 23 055009, (2013) 
[8] F. Zamkotsian, P. Lanzoni, E. Grassi, R. Barette, C. Fabron, K. Tangen, L. Valenziano, L. Marchand, L. Duvet "Successful evaluation for space applications of the 2048x1080 DMD," in Proceedings of the SPIE conference on MOEMS 2011, Proc. SPIE 7932, San Francisco, USA (2011)

[9] F. Zamkotsian, P. Spano, L. Martin, M. Riva, P. Lanzoni, F. Zerbi, L. Valenziano, L. Nicastro, "DMD-based MOS demonstrator on Galileo telescope," in Proceedings of the SPIE conference on Astronomical Instrumentation 2010, Proc. SPIE 7735, San Diego, USA, (2010)

[10] Frederic Zamkotsian, Harald Ramarijaona, Manuele Moschetti, Patrick Lanzoni, Marco Riva, Nicolas Tchoubaklian, Marc Jaquet, Paolo Spano, William Bon, Romain Alata, Luciano Nicastro, Emilio Molinari, Rosario Cosentino, Adriano Ghedina, Manuel Gonzalez, Walter Boschin, Paolo Di Marcantonio, Igor Coretti, Roberto Cirami, Filippo Zerbi, Luca Valenziano, "Building BATMAN: a new generation spectro-imager on TNG telescope ", in Proceedings of the SPIE conference on Astronomical Instrumentation 2016, Proc. SPIE 9908, Edinburgh, United Kingdom, (2016)

[11] Frederic Zamkotsian, Patrick Lanzoni, Nicolas Tchoubaklian, Harald Ramarijaona, Manuele Moschetti, Marco Riva, Marc Jaquet, Paolo Spano, William Bon, Mathieu Vachey, Luciano Nicastro, Emilio Molinari, Rosario Cosentino, Adriano Ghedina, Manuel Gonzalez, Walter Boschin, Paolo Di Marcantonio, Igor Coretti, Roberto Cirami, Filippo Zerbi, Luca Valenziano, " BATMAN@ @NG: Instrument integration and performance", in Proceedings of the SPIE conference on Astronomical Instrumentation 2018, Proc. SPIE 10702, Austin, USA, (2018)

[12]F. Zamkotsian, O. Ilbert, J. Zoubian, A. Delsanti, S. Boissier, A. Lancon, " BATMAN flies: a compact spectroimager for space observation," in Proceedings of the SPIE conference on Astronomical Instrumentation 2014, Proc. SPIE 9143, Montreal, Canada, (2014)

[13] Mathieu Vachey, Frédéric Zamkotsian, Vincent Costes, Hervé Benard, Arnaud Liotard, Nicolas Tetaz, "New spectro-imager designs for MOEMS-based instruments in Earth and Universe observation," Proc. SPIE 11483, Novel Optical Systems, Methods, and Applications XXIII, 1148308 (2020); doi: 10.1117/12.2569670

[14] Frederic Zamkotsian, Igor Zhurminsky, Patrick Lanzoni, Nicolas Tchoubaklian, Christian Schneider, Sören Fricke, Marc Schnieper, Fabian Lütolf, "Convex blazed gratings for high throughput spectrographs in space missions," in Proceedings of the International Conference on Space Optics (ICSO) 2018, Chania, Greece, Proc. SPIE 11180, (2019); doi.org/10.1117/12.2536100

[15] M. Zerrad, M. Lequime, S. Liukaityte and C. Amra, "Parasitic light scattered by complex optical coatings: modelisation and metrology," CEAS Space Journal, (2017)

[16] M. Fouchier, M. Zerrad, M. Lequime and C. Amra, "Wide-range wavelength and angle resolved light scattering measurement setup," Optics Letters, vol. 45, pp. 2506-2509, (2020) 